\title{
Dentoalveolar surgery and Prevention of Medication-related Osteonecrosis of the Jaw (MR-ONJ) in patients treated with Bisphosphonates: our experience.
}

\author{
Fabio Lonardi ${ }^{1}$, Giorgia Capocasale ${ }^{1}$, Francesca Zotti ${ }^{1}$, Riccardo Nocini ${ }^{1}$, Annarita Signoriello ${ }^{1}$, giorgio panozzo, \\ Massimo Albanese ${ }^{1}$ \\ 1 University of Verona
}

Funding: The author(s) received no specific funding for this work.

Potential competing interests: The author(s) declared that no potential competing interests exist.

\section{Abstract}

\begin{abstract}
:
Bisphosphonates (BPs) are the most commonly used antiresorptive drugs to prevent skeletal-related events (SREs) in cancer and osteometabolic patients. A potential adverse drugs reaction associated with the administration of these drugs is Medication-related Osteonecrosis of the Jaw (MR-ONJ); its frequency in cancer patients is higher than in osteometabolic patients.

Different risk factors have been identified: drug type, duration of treatment, demographic, systemic and local factors. Among the latter, the most reported and investigated are dentoalveolar surgical procedures, anatomical factors, prosthesis and associated local oral inflammatory diseases.

To date, MR-ONJ pathophysiology remains unclear, therefore, definitive protocols on prevention in oral surgery have not been established yet.

Several studies had investigated the procedures to prevent the occurrence of MR-ONJ after oral surgery, particularly for tooth extraction. These proposed protocols included antibiotic and mouthwash treatment, atraumatic extraction, primary closure of the extraction socket, laser biostimulation and the use of autologous platelet concentrates.

The present study aimed to evaluate our one year experience in the prevention of MRONJ after dentoalveolar surgery in patients receiving bisphosphonate therapy. The protocol described and proposed by the Italian Society of Oral and Maxillofacial Surgery (SICMF) and the Italian Society of Oral Pathology and Medicine (SIPMO) has been followed; this protocol includes medical prophylaxis with strictly described surgical procedures
\end{abstract}

Background: Bisphosphonate are commonly used to prevent skeletal related events in cancer and osteometabolic patients ${ }^{[1]}$. ONJ is a possible adverse drug reaction related to the use of these drugs in patient who undergo oral surgery while taking bisphosphonates. To treat $\mathrm{ONJ}$ in early stages minor surgery is required ${ }^{[2]}$, however if not treated properly the disease could spread leading to major surgery and extensive bone resection ${ }^{[3][4]}$. Since many patients affected by ONJ are old, they may be unfit for major surgery procedure, or unwilling to do so, therefore conservative treatments have been 
proposed $^{[5]}$, although they are not widespread.

Different risk factors have been identified, the most reported are oral surgical procedures. Different research teams have investigated the disease and proposed prevention protocols, however definitive protocols have not been established yet. Oral and maxillofacial surgeon routinely perform surgery on patients with history of bisphosphonate therapy and/or active use of bisphosphonates both for prevention and treatment of $\mathrm{ONJ}$; however since no defined protocols have been established results may vary. It must also been taken into account that there could be other factors influencing the outcome of the surgery and the incidence of ONJ such as the experience of the surgeon, which have not been thoroughly investigated.

Materials and Methods: This research reviewed patients with history of bisphosphonates use or active use of bisphosphonates who underwent oral surgery procedure in the oral and maxillofacial surgery unit of Policlinico GB Rossi in Verona between 1/1/2019 and 31/12/2019. An excel (Microsoft ${ }^{\circledR}$ ) database was created, demographic data were collected as well as data regarding comorbidities, type of drug and duration of treatment. We excluded patients with an history of radiation therapy to the craniofacial region.

To perform the tooth extraction, protocols described and proposed by the Italian Society of Oral and Maxillofacial Surgery (SICMF) and the Italian Society of Oral Pathology and Medicine (SIPMO) were followed ${ }^{[6]}$.

Results: A total of 43 patients were selected, 31 (72.09\%) female and $12(27.91 \%)$ male, average age was 67,98 12,33 years. 9 patients were treated with alendronate, 1 with neridronate, 4 with clodronate, 3 with ibandronate, 15 with zoledronate, 5 with risedronate, 3 had history of multiple bisphosphonate over the years, 3 patients reported the use of bisphosphonate however they could not recall the name and/or did not produce the documentation.

29 patients used bisphosphonate to treat osteoporosis, 10 patients used bisphosphonate to treat malignat neoplasms, 4 used bisphosphonate for other reasons.

10 patients had active neoplasms: 2 multiple Myeloma, 2 breast cancer, 6 other neoplasms; 2 had history of previous breast cancer, however at the time of surgery there was no evidence of relapse.

At present patients are still in follow up, up to date there is not evidence of ONJ onset due to oral surgery.

\section{Conclusions:}

Oral surgery is generally a safe procedure even in patients with history of bisphosphonate use. Following perioperative protocols ensures successful surgery and seems to provide good protection against ONJ. In the present study surgeries were performed by doctors of various level of experience (both residents and trained surgeons), however, out of 43 patients none developed ONJ after surgery. This suggests that SIPMO protocols, although not yet internationally approved, are an effective mean to prevent ONJ onset. Moreover the present abstract suggests that the effect seems to be independent from the skill of the surgeon. Further studies on lager samples are required to validate these results; ONJ is a relatively "new" disease, however due to the growing prescription of bisphosphonates a consensus conference to define international treatment protocols has not been held yet. 


\section{References}

1. 'Katlynn M. Mathis, Kathleen M. Sturgeon, Renate M. Winkels, Joachim Wiskemann, et al. (2018). Bone resorption and bone metastasis risk. Medical Hypotheses, vol. 118, 36-41. doi:10.1016/j.mehy.2018.06.013.

2. 'Amerigo Giudice, Selene Barone, Federica Diodati, Alessandro Antonelli, et al. (2020). Can Surgical Management Improve Resolution of Medication-Related Osteonecrosis of the Jaw at Early Stages? A Prospective Cohort Study. Journal of Oral and Maxillofacial Surgery, vol. 78 (11), 1986-1999. doi:10.1016/j.joms.2020.05.037.

3. ^Alberto Bedogni, Giorgia Saia, Giordana Bettini, Anita Tronchet, et al. (2011). Long-term outcomes of surgical resection of the jaws in cancer patients with bisphosphonate-related osteonecrosis. Oral Oncology, vol. 47 (5), 420 424. doi:10.1016/j.oraloncology.2011.02.024.

4. ' P. Procacci, M. Albanese, L. Trevisiol, V. Favero, et al. (2017). Medication-related osteonecrosis of the posterior maxilla: surgical treatment using a combined transnasal endoscopic and intraoral approach, our experience with seven consecutive patients. Clin Otolaryngol, vol. 43 (2), 685-691. doi:10.1111/coa.12999.

5. 'Massimo Albanese, Francesca Zotti, Giorgia Capocasale, Stefano Bonetti, et al. (2020). Conservative non-surgical management in medication related osteonecrosis of the jaw: A retrospective study. Clin Exp Dent Res, vol. 6 (5), 512518. doi:10.1002/cre2.303.

6. 'Olga Di Fede, Vera Panzarella, Rodolfo Mauceri, Vittorio Fusco, et al. (2018). The Dental Management of Patients at Risk of Medication-Related Osteonecrosis of the Jaw: New Paradigm of Primary Prevention. BioMed Research International, vol. 2018 , 1-10. doi:10.1155/2018/2684924. 